\title{
Celiac disease presenting with recurrent pyrexia and lobular panniculitis: a case report and review of literature
}

\author{
Tunde Maiyaki Ibrahim ${ }^{1,2 *}$, Abdullah Al-allawi ${ }^{1}$, Robert Henry Feiler ${ }^{3}$ and Ritoo Chowdary ${ }^{1}$ \\ ${ }^{1}$ Department of Medicine, Goulbourn Valley Base Hospital, Graham Street, Shepparton Vic 3630, Australia \\ ${ }^{2}$ The University Of Melbourne, Rural Clinical school Of Medicine, Graham Street, Shepparton Vic 3630, Australia \\ ${ }^{3}$ Departemnt of Medicine, The Alfred Hospital, Commercial Street, Melbourne, Vic, Australia
}

\begin{abstract}
We describe the case of a 27 yrs old Indian lady who present with relapsing fever, subcutaneous nodules with erythematous plaque on the left forearm and transient diarrhea. She was found to have hypothyroidism, and CT scan of the chest, abdomen and pelvic show multiple skin nodules. Biopsy of the erythematous forearm lesion revealed lobular panniculitis. Anti- transglutaminase and deamidated gliadin antibodies were positive and duodenal biopsy revealed celiac disease. Screening for Parasitic, infectious, connective tissue diseases and vasculitis where all negative. The lobular panniculitis and fever responded excellently to a 4 weeks tapering course of corticosteroid and gluten free diet. In conclusion this case revealed an atypical presentation of celiac disease and it association with lobular panniculitis.
\end{abstract}

\section{Background}

Classical celiac disease (CCD) is dominated by gastrointestinal (GTI) symptoms while the atypical celiac diseases (ACD) is associated with extra-intestinal symptoms [1]. The extra-intestinal symptoms include various dermatological conditions the most common ones are dermatitis hepetiformis, linear IgA bullous dermatosis (LABD), urticaria, hereditary angioneurotic edema(HAE), leucocytoclastic vasculitis, erythema nodosum (EN), erythema elevatum diutinum (EED), necrolytic migratory erythema (NME), psoriasis, vitiligo disease, dermatomyositis, and oral lichen planus [2]. lobular panniculitis (LP) has not been described in association with celiac disease to the knowledge of the authors therefore we present this case of ACD with an unusual dermatological manifestation so that clinician will henceforth consider $\mathrm{CD}$ as a possible diagnosis in patients with fever and skin lesions.

\section{Case report}

We present a 27 yrs old woman migrant from India with background history of closed ASD as a child who presented with relapsing high grade fever for about 4 months and transient diarrhea. She had similar febrile episodes associated with mildly tender left forearm indurated lesion about 2 months back while in India which resolved spontaneously and no obvious cause was found despite extensive investigations. Examination revealed multiple subcutaneous nodules in the neck, anterior abdominal wall, upper limbs and multiple breast fibroadenomatas. By day 3 of admission an erythematous underrated lesion was observed in the dorsal aspect of the left forearm close to the wrist. The punch biopsy of this lesion revealed lobular panniculitis with subcutaneous fat layer infiltration by neutrophils, lymphocytes and histocytes with no vasculitis (Figures 1 and 2). Full blood count revealed microcytic anemia secondary to iron deficiency and CRP was elevated at $152 \mathrm{mg} / \mathrm{l}$ suggestive of ongoing inflammation. Other markers of inflammation beta -2- macroglobulin (3.27 umg/l), ANA (1:640 with speckle pattern), ANCA II F were also elevated. Other specific investigations for connective tissue disease and vasculitis were negative. Peripheral blood film flow cytometry done to exclude lymphoproliferative disease showed polyclonal B lymphocytes abnormality with CD 19+, CD 22+, CD 23+, and FMC 71 with a shift in kappa:lamba of $3: 1$ in $25 \%$ of the lymphocytes feature consistent with an inflammatory condition.

Three blood cultures and a stool culture were negative. The renal and liver functions were also normal expect for mildly elevated AST of 32 IU/l. Thyroid function was consistent with hypothyroidism with TSH of $12.18 \mathrm{Mu} / \mathrm{L}$ and FT3 of $3.0 \mathrm{pmol} / \mathrm{l}$. Malaria antigen

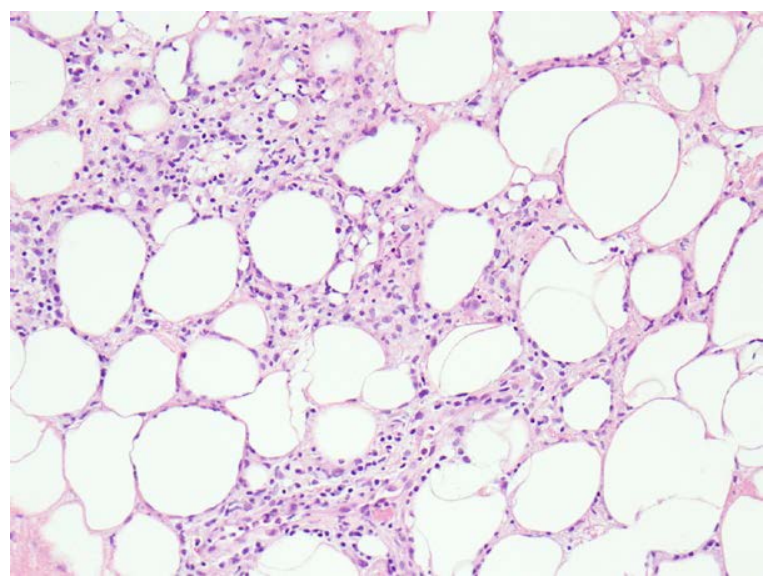

Figure 1. Histology of the punch -biopsy of the patient's skin showing mixed inflammatory cells (lymphocytes,neutrophils and histocytes) infiltrate of the adipose of the subcutis consistent with diagnosis of lobular panniculitis.

Correspondence to: Tunde Maiyaki Ibrahim, Department of Medicine, Goulbourn Valley Base Hospital, Graham Street, Shepparton Vic 3630, Australia, E-mail: imaiyaki@yahoo.com

Key words: celiac disease, lobular panniculitis, extra-intestinal manifestation, autoimmune disease

Received: May 14, 2017; Accepted: May 25, 2017; Published: May 29, 2017 


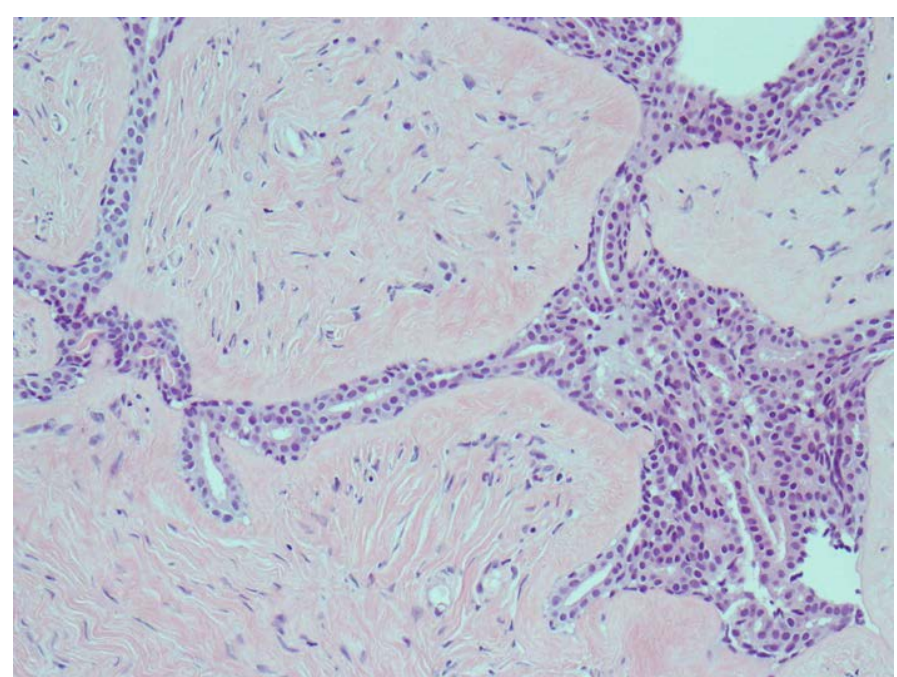

Figure 2. Histology of patient's adiposesubcutis with infiltration by lymphocytes, neutrophils and histocytes with small amount of fibrosis surrounding the inflamed fat consistent with lobular panniculitis.

and interferon gamma release assay tests were negative. She had transesophageal echocardiography with no futures of endocarditis. Tazocin and vancomycin were given for presumed sepsis. With no remittance in the fever and all cultures been negative after $72 \mathrm{hrs}$ the antibiotics were discontinued. As no obvious cause of the relapsing fever and lobular panniculitis was found despite extensive investigation we made a diagnosis of idiopathic lobular panniculitis and started treatment with prednisolone $50 \mathrm{mg}$ daily. She responded dramatically to the steroid which she had for about 4 weeks. However in view of the iron deficiency anemia and diarrhea we screened serologically for celiac disease with anti-transglutaminase and deamidated gliadin antibodies both of which were positive. She then went to have gastroscope and the duodenal biopsy histology revealed subtotal villous atrophy, broad blunted villi with increase intraepithelial lymphocytes consistent with the diagnosis of coeliac disease. She started on gluten free diet and thyroxine was given for the hypothyroidism. Review 5 months post her presentation she has not had any relapse of the fever, diarrhea or skin lesion.

\section{Discussion}

$\mathrm{CD}$ is an immunopathological inflammatory disease precipitated by intestinal exposure to gluten present in wheat, barley and oat in those who are genetically predispose, with a worldwide incidence estimated to be $0.5-3 \%$ in the adult population $[1,3]$. CD does present with wide spectrum of gastrointestinal and extra-intestinal symptoms including lots of dermatological conditions [3,4]. Fernandez et al. [4], in their study observed that almost $50 \%$ of pateints had ACD and GIT symptoms were absent in about 33\%. Gastrointestinal and malabsorption syndromes especially of iron, vitamin D, folate and other trace elements and vitamins usually absorbed from the upper small intestine are common in CCD while in the ACD the extraintestinal symptoms predominate $[1,5]$. The case presented conform with this finding as she has atypical presentation with fever and an unusual skin manifestation as major symptom. This is similar to the case reported by Cooney and El-Matary [6], of a patient with CD whose main features at presentation was also fever. Therefore unexplained fever should raise the possibility of CD especially in the presence of common dermatological conditions such as dermatitis hepetiformis, linear IgA bullous dermatosis (LABD), urticaria,hereditary angioneurotic edema (HAE), leucocytoclastic vasculitis, erythema nodosum (EN), erythema elevatum diutinum (EED), necrolytic migratory erythema (NME), psoriasis, vitiligo disease, dermatomyositis, and oral lichen planus [2,7], and as this case demonstrated LP. The association between CD and LP can be explained by the fact that both conditions have immunopathological basis. The immunopathology of LP is revealed in the 3 histopathological stages described on skin biopsy in LP. The first stage is characterized by acute inflammation with fat lobules infiltration by neutrophils, lymphocytes and histocytes. This is similar to intestinal mucosal infiltration by lymphocytes seen in $\mathrm{CD}$. In the second stage foam cell are the cardinal features with lots of macrophages phagocytosing dead fat cells while in the third stage the foam cells are replaced by fibroblasts and fibrous tissue $[2,8]$.

The combination of CD, LP and autoimmune hypothyroidism in this case like in earlier studies show that $\mathrm{CD}$ is a clinical condition that can be associated with most other autoimmune diseases. Earlier studies have demonstrated these association between CD and common autoimmune diseases such as type $1 \mathrm{DM}$, SLE, and thyroid diseases $[9,10]$. Autoimmune and immunological diseases such as SLE, lymphoproliferative diseases, pancreatitis, sarcoidosis, histocytic cytophagic panniculitides and alpha 1 anti-trypsin deficiency state have also been report in association with LP [2].

\section{Conclusion}

This case further illustrates that $\mathrm{CD}$ is an autoimmune diseases that can be associated with any other immune-pathologically based disease such as LP and thyroid diseases. And should be considered in any patients presenting with nonspecific symptoms on the background of another autoimmune disorder.

\section{References}

1. Gasbarrini G,Malandrino N, Giorgio V,Fundaro C, Cammarota G, et al. (2008) Celiac disease : what's new about it? Dig Dis 26:12-17. [Crossref]

2. Abenavoli L, ProiettiI, LeggioL, FerrulliA, VonghiaL, et al. (2006) Cutaneous manifestations in celiac disease. World J Gastroenterol12: 843-852.

3. Kang JY, Kang AH, Green A, Gwee KA, Ho KY (2013) Systematic review: worldwide variation in the frequency of coeliac disease and changes over time. Aliment Pharmacol Ther 38: 226-245.[CrossRef]

4. Fernandez Salazar LI, de la Torre Ferrera N,Velayos Jimenez B,Nocito Colon M, Gonzalez Hermandez JM, et al. (2008) Diagnostic problems in adult celiac disease.Rev EspEnfem Dig 100:24-28. [Crossref]

5. Capristo E, Addolorato G, Mingrone G, De Gaetano A, Greco AV, et al. (2000) Changes in body composition, substrate oxidation, and resting metabolic rate in adult celiac disease patients after a 1-y gluten-free diet treatment.Am J Clin Nutr 72:76-81. [Crossref]

6. Cooney MJ, El-Matary W (2013) Celiac disease presenting as Fever of unknown origin. Case Rep Gastrointest Med 2013: 676327.[CrossRef]

7. Zone JJ (2005) Skin manifestations of celiac disease. Gastroenterology128: S87-91. [CrossRef]

8. Cooper BT, Holmes GK, Cooke WT.Coeliac disease and immunological disorders. $\mathrm{Br}$ Med J1: 537-9.[Crossref]

9. Sun X, Lu L, Yang R, Li Y, Shan L, et al. (2016) Increased Incidence of Thyroid Disease in Patients with Celiac Disease: A Systematic Review and Meta-Analysis. PLoS One 11:e0168708. [Crossref]

10. Roy A, Laszkowska M, Sundström J, Lebwohl B, Green PH, et al. (2016) Prevalence of Celiac Disease in Patients with Autoimmune Thyroid Disease: A Meta-Analysis. Thyroid26: 880-890.[CrossRef]

Copyright: C2017 Ibrahim TM. This is an open-access article distributed under the terms of the Creative Commons Attribution License, which permits unrestricted use, distribution, and reproduction in any medium, provided the original author and source are credited. 